\title{
MANIPULATIVE SURGERY.
}

\author{
By R. C. ELMSLIE, O.B.E., M.S., F.R.C.S. \\ (Orthopadic Surgeon, St. Bartholomew's Hospital.)
}

In one sense all surgery is manipulative. The reduction of dislocations, the setting of fractures, the correction of deformities and the manipulations carried out during an operation, all constitute the handicraft of surgery, and it is only by a convention that the name Manipulative Surgery is applied to certain special procedures. In the sense in which it is used here, manipulative surgery means the manipulation of joints and muscles as a method of overcoming stiffness of movement, disability or pain. Manipulations to correct major deformities, such as a club foot or a congenital dislocation of the hip, are omitted. Even in this restricted sense manipulations have been used in surgery over a very long period, although it would appear that until comparatively recently manipulations were perhaps more often carried out by unqualified bonesetters and surgical mechanicians who were also employed in the making of splints and appliances. This was certainly the case during the eighteenth century, although certain surgeons even at that time made use of manipulations, and it is recorded that in I782 Hey manipulated a knee joint for an injury to a semilunar cartilage.

Coming to more recent times, it is as well to realize that during the second half of the nineteenth century the treatment of all sorts of joint diseases and injuries was very considerably influenced by the teaching of Hilton and $\mathrm{H}$. O. Thomas both of them advocates of rest and immobilization. In fact, Hilton's teaching. that pain indicated the necessity for rest, and Thomas's fear of moving an inflamed joint, were undoubtedly responsible for preventing the use of manipulations, movements and exercises in surgery and for delaying the adoption of manipulative methods by the qualified practitioner. During this period the unqualified bonesetter met with a very considerable element of success, his chances being favoured by the stiff joints which resulted from the more regular forms of treatment then in vogue. These successes of the bone-setter were investigated by Sir James Paget and his pupil, Howard Marsh, the latter of whom used to include a lecture on bone setting in his annual course of lectures at St. Bartholomew's. But although these two surgeons did a good deal to introduce methods of manipulation so that these methods have actually been in use at St. Bartholomew's for over forty years, manipulative surgery owes a much greater debt to Wharton Hood, who actually worked with Hutton, the best known bone-setter of his day, and introduced his methods into surgery. It is unfortunate that Wharton Hood was not very well received by his surgical colleagues and held no teaching position, so that his methods did not obtain any wide publicity.

However, since the end of the nineteenth century there has been a steady tendency amongst the surgical profession to use methods of manipulation more and more, using manipulation in a rational manner, to try and ease stiff joints and to stretch ligaments and muscles that are shortened, thus restoring normaI functional use. But a new complication has arisen; Still's introduction of osteopathy brought forward the suggestion that manipulations can do a great deal more than this. He introduces the theory that some little displacement in the spine can produce symptoms of a major illness and that the correction of such displacement by manipulation can cure, for example, epilepsy, gastric ulcer or an acute infection such as diphtheria. Osteopathy has not had the field to itself. It has been followed by such variants as chiropractic, natureopathy, etc., each with their own 
theories of the results of manipulation and with accessory treatment thrown in. Moreover, these cults have tended to absorb the manipulations formerly carried out by the bone-setter and the surgeon, so that the osteopath may be at one time manipulating the spine for some general disease, at another manipulating the ankle for some adhesions due to a sprain and at another reducing a displaced cartilage in the knee joint. In this way surgery, bone-setting, osteopathy, etc. have become inextricably mixed.

The development of manipulative methods in surgery has perhaps been slow but it is to be hoped that it has grown up in a rational manner based on a knowledge of pathology, and in fact the one thing claimed for surgical manipulation would be that it is carried out after a proper investigation of the underlying pathological condition and that its methods are based upon anatomy, physiology and pathology. Such surgical manipulations may be divided into two categories. First, those major manipulations which have to be carried out whilst the patient is under an anæsthetic and second, the minor manipulations which are usually carried out by the massage profession in the course of their treatment. These minor manipulations must also be based upon a sound pathology. They are often called passive movements, but they are not by any means always passive. Quite frequently the masseur has to manipulate a joint which is decidedly stiff, and in which movement is much resisted. Before even such mild manipulations are carried out an estimate of the exact underlying condition should be formed by the surgeon and this information should be transmitted to the masseur.

\section{Manipulation of Stiff Joints.}

The most obvious case requiring manipulation is that in which a joint such as the hip or knee has been left stiff as a result of either injury or inflammation. The first step is then the pathological diagnosis. By means of the history, including the nature of any injury and the course of any inflammatory disease, the clinical condition, and the X-ray appearance, a judgment must be formed as to exactly what factors are hindering movement. For example, in the case of an old fractured femur, stiffness of the knee may be due to the formation of fibrous adhesions in the quadriceps muscle or possibly to the patella being bound down. Or there may be changes in the area around the knee joint; the capsule of the joint may be thickened, the loose tissue which lies between the capsule and the synovial membrane may be fibrotic, or the synovial membrane itself may be adherent and its normal folds and pockets diminished in size or abolished. If the case has been one of an inflammatory lesion of the knee, there may be further changes visible in the radiogram-roughening or irregularity of the articular surface or the formation of bone which might mechanically block the movement. When a judgment has been formed on the pathological condition, an idea can be obtained of the probable benefit that will result from manipulation; for example, a fibrosed quadriceps can be gradually stretched, the manipulation being taken slowly and not carried very far at one sitting. The same applies to a patella which is partly, but not completely, fixed and also to thickening of the capsule and moderate adhesions of the synovial membrane. But if the joint surfaces have been much altered and are irregular, manipulation must be purely experimental and is rather a test to see whether movement is possible than an attempt to improve the movement at one sitting. If the movement is blocked by the formation of bone, manipulation is obviously contra-indicated. The most important manipulations for this type of stiffness are those of the major joints which have been stiffened as a result of fractures in their neighbourhood or as a result of some infective 
lesion from which recovery has taken place. Examples are, manipulation of a $\subseteq$ stiff knee, of a hip at certain stages of osteo-arthritis, and a painful stiff shoulder $\overrightarrow{\vec{\sigma}}$ with much loss of movement, following upon injury without fracture or a sub-acute $-\frac{-}{\circ}$ form of arthritis. The performance of manipulations of this class is nothing new in surgery, but the decision as to the advisability of manipulation and the benefit that will probably be derived is always a very difficult one.

In this case, as in fact in the majority of cases of surgical manipulation, a $\%$ full and complete anæsthesia is desirable so that the patient is quiet, the muscles $\overrightarrow{0}$ relaxed and the surgeon not hurried in his work. It is most important not to $\overrightarrow{ }$ carry the manipulation of a really stiff joint too far at one sitting, as this may $\omega_{\circ}$ result in a very considerable local reaction with much swelling and pain which hinders further treatment. It is wiser to try and free the joint by more gradualö methods of repeated manipulations with physical treatment carried out in the $\vec{\omega}$ intervals.

\section{Manipulation for Minor Adhesions.}

Whereas these major manipulations are a very obvious form of treatment, the necessity for manipulation in minor adhesions may not be recognized by someone $\stackrel{ }{5}$ unaccustomed to treating the condition. For example, a knee is uncomfortable $\vec{\circ}$ or weak or gives way occasionally and an ordinary examination fails to disclose ? any abnormality, but absolutely full flexion and rotation in a flexed position may ${ }_{\mathbb{D}}$ be slightly restricted and give rise to pain. Such a knee will very probably recover ${ }^{\stackrel{D}{-}}$ completely after being put through its full movements under an anæsthetic and $\vec{\oplus}$ having some simple exercise treatment afterwards. The same thing applies 1.0 the ankle in which a slight restriction of inversion remains after a sprain and 0 a wrist in which on forced flexion there is pain in the region of the tendons of the carpal extensors. Similar minor adhesions may occur in the shoulder and in fact in any other joint. In the feet and toes some slight loss of movement is $\frac{\mathrm{O}}{\mathbb{D}}$ quite commonly a cause of disability, and manipulation of the tarsal joints and $\stackrel{D}{\vec{D}}$ of toes that are contracted up, followed by a suitable physical treatment, will often $\vec{\overrightarrow{ }}$ render a chronically painful foot comfortable. The manipulation of a tennis elbow may perhaps come in this class, but its pathology is uncertain and has given rise to a great deal of controversy. Not all tennis elbows are cured by manipulation; in some there is a toxic factor which must be eliminated before the elbow will 3 recover and in some there is a definite arthritis in the elbow joint. But if these two conditions are eliminated, a chronic tennis elbow will probably be cured by manipulation if it reacts to the following test of movement:- The elbow is extended fully, the forearm pronated and the wrist flexed. With one hand grasping the arm above the elbow and the other grasping the hand, these three movements are 9 forced and the patient winces because of the pain felt in the region of the head of $\frac{D}{0}$ the radius. Under an anæsthetic the elbow is first flexed up fully and the limb grasped in the above manner, the thumb of the hand which is holding the region $N$ of the elbow being pressed against the back of the head of the radius. Extension $N$ of the elbow, pronation of the forearm and flexion of the wrist are then forced N simultaneously, the head of the radius being pushed forwards with the thumb. This manipulation can be followed by graduated exercises to get back strength ine the forearm muscles.

\section{Muscular Adhesions.}

It is possible that the manipulation for tennis elbow is really an instance of $\overrightarrow{0}$ stretching muscular adhesions, but there may be an underlying small synovial $\stackrel{\oplus}{\circ}$ pouch which is also pulled open. But muscular pain occurs quite definitely in $\stackrel{\vec{\curvearrowright}}{\circ}$ 
the calf after minor injuries, and in the spinal muscles. In the calf, manipulation simply consists of forceful dorsiflexion to stretch the calf muscles. In the back, muscular adhesions are undoubtedly one cause of backache, but this condition is dealt with separately in this series.

\section{Subluxations of Joints and Cartilages.}

In certain forms of stiff neck there is an actual subluxation of one side between the articular processes of two of the vertebræ, usually about the level of the third or fourth. Such a subluxation can occur as a result of muscular action and has even been known to occur during sleep. In a subluxation on the left side the head is a little tilted and rotated towards the right and there is local pain and possibly a slight fullness to be felt in the middle of the neck on the left side. Manipulation under an anæsthetic, pulling the head, tilting further to the right and then rotating to the left, will probably result in an immediate easing of the movement with a full recovery after a few days. It is alleged that in certain cases of low backache there is a subluxation of the sacro-iliac joint. Undoubtedly many of these cases are cured by manipulation, but proof of the existence of a subluxation is lacking. Displacements of intra-articular cartilages occur in the knee and in the tempero-mandibular joint and in both of these the symptoms can be relieved and the condition possibly cured by a manipulation. In the knee joint, the condition which most frequently necessitates manipulation is locking of the joint in a position of flexion with pain over the anterior part of the internal cartilage. There is some misconception as to the meaning of locking of the knee joint. This does not mean that movement in the knee is abolished, but simply that full extension is blocked as a result of something which happens quite suddenly. Needless to say, before manipulating it is advisable to take X-ray photographs to make sure that there is no bony loose body, because if there is, manipulation is likely to do further damage.

Manipulation for an internal semilunar cartilage is carried out as follows:The patient is under full anæsthesia. The thigh is grasped with one hand and the ankle and foot with the other. The knee is then flexed fully, the foot being rotated out. At this stage (that is, in full flexion) the hand may be removed from the foot and placed under the head of the tibia which is lifted forcibly forwards from the femur. The foot is then again grasped and the knee rapidly and fully extended, whilst pressure is made against the outer side of the knee joint and the toot is rotated out. If this mancuvre fails to produce full extension it is tried again, the foot.being rotated in as the knee is extended and, if it still fails, the knee may be brought gradually down from a position of flexion to a position of full extension whilst the leg is rotated in and out several times during the movement. The first manouvre described is that which was given by Howard Marsh in his book, "Diseases of the Joints" in 1886.

Put quite briefly, it may be said that manipulation of the knee in the case of an internal cartilage injury will usually, but not always, render the knee immediately comfortable. Probably in such cases the cartilage is either restored to its normal position or has been forced into the middle of the joint where there is sufficient room for it. But sometimes full extension is not obtained and then it is usually desirable to advise operative treatment. In a displacement of the external cartilage one may similarly get an immediate complete success, but often one only gets an improvement. 
Manipulation for an injury of the internal cartilage sometimes results in permanent cure of the condition; that is to say the cartilage does not become again displaced or lock the joint, but this is not anything more than a chance, and no promises of a complete cure can be givcn. Displacement of the external cartilage is practically never permanently cured by manipulation.

A tempero-mandibular joint may also become locked because of displacement of its intra-articular cartilage. When it does so, the jaw cannot be opened fully or easily and there is a feeling that it is being pushed towards one side. Manipulation is carried out by opening the jaw with a gag whilst the patient is under an anæsthetic and then lifting the chin in order to open up the region of the joint.

\section{Manipulation of Nerves.}

The only nerve that is commonly manipulated is the sciatic, which is stretched under an anæsthetic by flexion of the hip with the knee extended. The theory behind this is that the roots of the lumbar or sacral nerves may have become slightly adherent in the inter-vertebral foramina and that possibly in an oldstanding case there may be some fibrous contraction in the nerve itself. At the present time such a stretching of the sciatic nerve is usually combined with manipulation of the lumbar and pelvic region.

In a short article it is only possible to lay down the principles upon which manipulations are based and to describe a few of the commoner conditions, but it may be again stated that all surgical manipulations should be based upon careful diagnosis and an estimate of the pathological conditions present. There is perhaps a tendency in the medical profession to be a little too careful and for this reason to fail to carry out a manipulation which can produce a dramatic cure. For this reason, it is as well to try and eliminate disease or serious injury and to be fully convinced that there is no underlying condition of importance. One may then feel free to carry out an experimental manipulation; for example, if some movement is a little painful when forced and we can find nothing to account for this pain, a manipulation to stretch the painful part will frequently cure the condition. It is perhaps the omission to treat rather trivial conditions in this way that has caused the medical profession to lose reputation in comparison with the unqualified manipulator. 